\title{
Distributed Higher Order Consensus Protocols in Multiagent Dynamical Systems
}

\author{
Wenwu Yu, Student Member, IEEE, Guanrong Chen, Fellow, IEEE, Wei Ren, Member, IEEE, Jürgen Kurths, and \\ Wei Xing Zheng, Senior Member, IEEE
}

\begin{abstract}
This paper studies general higher order distributed consensus protocols in multiagent dynamical systems. First, network synchronization is investigated, with some necessary and sufficient conditions derived for higher order consensus. It is found that consensus can be reached if and only if all subsystems are asymptotically stable. Based on this result, consensus regions are characterized. It is proved that for the $m$ th-order consensus, there are at most $\lfloor(m+1) / 2\rfloor$ disconnected stable and unstable consensus regions. It is shown that consensus can be achieved if and only if all the nonzero eigenvalues of the Laplacian matrix lie in the stable consensus regions. Moreover, the ratio of the largest to the smallest nonzero eigenvalues of the Laplacian matrix plays a key role in reaching consensus and a scheme for choosing the coupling strength is derived. Furthermore, a leader-follower control problem in multiagent dynamical systems is considered, which reveals that to reach consensus the agents with very small degrees must be informed. Finally, simulation examples are given to illustrate the theoretical analysis.
\end{abstract}

Index Terms-Algebraic graph theory, consensus region, higher order consensus, leader-follower control, multiagent system.

\section{INTRODUCTION}

$\mathbf{C}$ OLLECTIVE behaviors in groups of autonomous mobile agents are commonly concerned issues for study due to the observation of animal group behaviors, such as flocking and swarming, and their wide applications in biological systems, sensor networks [36], unmanned air vehicle (UAV) formations,

Manuscript received May 29, 2010; revised August 26, 2010 and November 17, 2010; accepted December 22, 2010. Date of publication February 10, 2011; date of current version July 27, 2011. This work was supported by the NSFC-HKRGC Joint Research Scheme under Grant N-CityU107/07, the DAAD Scholarship (Deutscher Akademischer Austauschdienst Dienst) under Ref. 423, the German Science Foundation (DFG) in the SFB 555, the National Science Foundation CAREER Award (ECCS-0748287), and a Research Grant from the Australian Research Council. This paper was recommended by Associate Editor H. Gao.

W. Yu is with the Department of Mathematics, Southeast University, Nanjing 210096, China and also with the School of Computing and Mathematics, University of Western Sydney, Penrith NSW 2751, Australia (e-mail: wenwuyu@gmail.com; wwyu@ seu.edu.cn).

G. Chen is with the Department of Electronic Engineering, City University of Hong Kong, Hong Kong, China (e-mail: eegchen@ cityu.edu.hk).

W. Ren is with the Department of Electrical and Computer Engineering, Utah State University, Logan, UT 84322 USA (e-mail: wren@engineering.usu.edu).

J. Kurths is with the Research Domain IV, Institute for Climate Impact Research, 14473 Potsdam, Germany, and also with the Institute for Complex Systems and Mathematical Biology, University of Aberdeen, Aberdeen AB24 3UE, U.K. (e-mail: Juergen.Kurths@ @ik-potsdam.de).

W. X. Zheng is with the School of Computing and Mathematics, University of Western Sydney, Penrith NSW 2751, Australia (e-mail: w.zheng@uws.edu.au).

Color versions of one or more of the figures in this paper are available online at http://ieeexplore.iee.org.

Digital Object Identifier 10.1109/TCSI.2011.2106032 robotic teams, etc. The study of collective behaviors aims to understand how cooperative group behaviors arise as a result of distributed local interactions among mobile individuals who only share information with their neighbors and simultaneously try to agree on certain global criteria of common interest.

Recently, much progress has been achieved in the study of collective behaviors of multiagent dynamical systems, such as consensus [4], [9], [10], [13], [18], [21]-[24], [33], [34], [36], synchronization [1], [5], [6], [14], [16], [17], [20], [27], [29], [31], [32], [35], [37], [38], and swarming and flocking [19], [25], [26]. Many investigations have been devoted to constructing conditions under which an agreement can be reached among a group of autonomous agents in a dynamically changing environment. In [26], Vicsek et al. proposed a simple discrete-time model to study a group of autonomous agents moving in the plane with the same speed but different headings, which by nature is a simplified version of the model introduced earlier by Reynolds to animate flocking behaviors [25]. Based on the algebraic graph theory [8], the study of Vicsek's model and its continuous-time counterpart has shown that consensus in a network with a dynamically changing topology can be reached if and only if the time-varying network topology contains a spanning tree frequently enough as the network evolves in time [4], [13], [18], [23].

Most existing reports on the consensus problem focus on the case where agents are governed by first-order dynamics [4], [13], [18], [23], [24] or second-order dynamics [9], [10], [21]. [22], [33], [34]. First-order consensus can be achieved if and only if the fixed network structure contains a directed spanning tree [13], [23]. In [22], it was shown that second-order consensus in linear multiagent systems can be reached if and only if the linear multiagent dynamical system has exactly two zero eigenvalues and all the other eigenvalues have negative real parts. However, for a general directed network, second-order consensus may fail even if the network contains a directed spanning tree [22]. In [33], some necessary and sufficient conditions for second-order consensus in multiagent dynamical systems with directed topologies were established. It was shown that both the real and imaginary parts of the eigenvalues of the Laplacian matrix of the corresponding network topology play key roles in reaching consensus.

Very recently, some higher order consensus algorithms in cooperative control of multiagent systems were studied in [24] based on the results derived in [22]. However, only third-order consensus was discussed there. In this paper, a general higher order consensus protocol is considered based on the transverse 
stability to the consensus manifold, which is originated from the study of synchronization in complex networks [27]. In [15], the notion of consensus region was first proposed to address the consensus problem in multiagent systems with general higher order dynamics based on observers design. However, in this paper, consensus in multiagent systems with classical higher order dynamics is considered and more detailed analysis about computing consensus regions is explicitly given for this particular consensus protocol. A detailed analysis of the higher order consensus algorithms is a prerequisite step to introduce more realistic dynamics into the model of each individual agent for future studies.

The main contribution of this paper is that some necessary and sufficient conditions are obtained for ensuring higher order consensus in multiagent dynamical systems and it is found that consensus can be reached if and only if all subsystems are asymptotically stable. Another contribution is a characterization of the stable consensus regions, and that consensus can be achieved if and only if all the nonzero eigenvalues of the Laplacian matrix lie in the stable consensus regions. It is also found that for the $m$ th-order consensus, there are at most $\lfloor(m+1) / 2\rfloor$ disconnected stable and unstable consensus regions. Moreover, it is shown that the ratio of the largest to the smallest nonzero eigenvalues of the Laplacian matrix plays a key role in reaching consensus and a scheme for choosing a corresponding coupling strength is derived. Furthermore, a leader-follower control problem in multiagent dynamical systems is considered, which reveals that to reach consensus the agents with very small degrees must be informed.

The rest of the paper is organized as follows. In Section II, some preliminaries on graph theory and model formulation are given. Some higher order consensus algorithms for multiagent dynamical systems are proposed in Section III. In Section IV, leader-follower control of multiagent systems is discussed, where each agent is governed by higher order dynamics. In Section $\mathrm{V}$, numerical examples are simulated to illustrate the theoretical analysis. Conclusions are finally drawn in Section VI.

\section{PRELIMINARIES}

In this section, some basic concepts and results about algebraic graph theory and model formulation are briefly introduced.

Let $\mathcal{G}=(\mathcal{V}, \mathcal{E}, G)$ be a weighted undirected network of order $N$, with a set of nodes $\mathcal{V}=\left\{v_{1}, v_{2}, \cdots, v_{N}\right\}$, a set of undirected edges $\mathcal{E} \subseteq \mathcal{V} \times \mathcal{V}$, and a weighted adjacency matrix $G=\left(G_{i j}\right)_{N \times N}$. An edge $\mathcal{E}_{i j}$ in the network $\mathcal{G}$ is denoted by the unordered pair of nodes $\left(v_{i}, v_{j}\right)$. By the definition of adjacency matrices for weighted graphs [11], weights $G_{i j}=G_{j i}>0$ are all positive if and only if there is an edge $\left(v_{i}, v_{j}\right)$ in $\mathcal{G}$. In this paper, only positively weighted networks are considered. A path between nodes $v_{i}$ and $v_{j}$ is a sequence of edges, $\left(v_{i}, v_{i_{1}}\right),\left(v_{i_{1}}, v_{i_{2}}\right), \ldots,\left(v_{i_{l}}, v_{j}\right)$, in the network with distinct nodes $v_{i_{k}}, k=1,2, \ldots, l$. An undirected network $\mathcal{G}$ is connected if there is a path between any pair of distinct nodes $v_{i}$ and $v_{j}$ in $\mathcal{G}, i, j=1,2, \ldots, N$.
Consider the following $m$ th-order dynamics in a multiagent system:

$$
\begin{aligned}
\dot{\xi}_{i}^{(1)}(t) & =\xi_{i}^{(2)}(t), \\
& \vdots \\
\dot{\xi}_{i}^{(m-1)}(t) & =\xi_{i}^{(m)} \\
\dot{\xi}_{i}^{(m)}(t) & =u_{i}, i=1,2, \ldots, N,
\end{aligned}
$$

where $\xi_{i}^{(k)} \in R^{n}$ are the states of the $i$ th node, which denotes the $(k-1)$ st derivative of $\xi_{i}^{(1)}, k=1, \ldots, l$, and $u_{i}$ is the control input. For notational simplicity, only $n=1$ is considered throughout the paper. One can easily generate the results to the case of $n>1$ by using Kronecker products [12].

Definition 1: The multiagent system (1) is said to achieve the $m$ th-order consensus if, for any well-defined initial conditions,

$$
\begin{aligned}
& \lim _{t \rightarrow \infty}\left\|\xi_{i}^{(k)}(t)-\xi_{j}^{(k)}(t)\right\|=0, \\
& k=1, \ldots, m ; i, j=1,2, \ldots, N .
\end{aligned}
$$

In order to achieve the $m$ th-order consensus, the following control input protocol is designed [24]:

$$
\begin{aligned}
u_{i}(t)=c \sum_{j=1, j \neq i}^{N} G_{i j} \sum_{k=1}^{m} \alpha_{k}\left(\xi_{j}^{(k)}(t)-\xi_{i}^{(k)}(t)\right) & \\
& i=1,2, \ldots, N
\end{aligned}
$$

where $c>0$ is the outer coupling strength, $\alpha_{k}>0$ are the inner coupling strengths, $G=\left(G_{i j}\right)_{N \times N}$ is the coupling configuration matrix representing the topological structure of the network and thus is the weighted adjacency matrix of the network, and the Laplacian matrix $L=\left(L_{i j}\right)_{N \times N}$ is defined by

$$
L_{i i}=-\sum_{j=1, j \neq i}^{N} L_{i j}, L_{i j}=-G_{i j}, i \neq j,
$$

which ensures the diffusion property that $\sum_{j=1}^{N} L_{i j}=0$.

Lemma 1: [11] The Lapacian matrix $L$ in an undirected network is symmetric and positive semi-definite. Moreover, $L$ has a simple eigenvalue 0 and all the other eigenvalues are positive if and only if the undirected network is connected.

Note that when $m=1$, the consensus algorithm (1), (2) reduces to the well-known first-order consensus protocol [13], [17], [18], [23], [27], [29], [31]-[33], [35], [37], [38]

$$
\xi_{i}^{(1)}(t)=-c \alpha_{1} \sum_{j=1}^{N} L_{i j} \xi_{j}^{(1)}(t), i=1,2, \ldots, N .
$$

Note also that, the control input (2) can be rewritten in a simpler equivalent form

$$
u_{i}(t)=-c \sum_{j=1}^{N} L_{i j} \sum_{k=1}^{m} \alpha_{k} \xi_{j}^{(k)}(t), i=1,2, \ldots, N .
$$

Throughout the paper, let $0=\lambda_{1} \leq \lambda_{2} \leq \cdots \leq \lambda_{N}$ be the $N$ eigenvalues of the Laplacian matrix $L$, and $1_{N} \in R^{N}$ $\left(0_{N} \in R^{N}\right)$ be a vector with all entries being $1(0)$. 


\section{THE $m$ TH-ORDER CONSENSUS IN A GENERAL FORM}

\section{A. Synchronization in Complex Networks}

Consider a complex dynamical network consisting of $N$ identical nodes with linearly diffusive coupling [17], [27], [29], [31], [32], [35], [37], [38]

$$
\dot{\eta}_{i}(t)=g\left(\eta_{i}\right)-c \sum_{j=1}^{N} L_{i j} D \eta_{j}(t), i=1,2, \ldots, N
$$

where $\eta_{i} \in R^{m}$ is the state vector of the $i$ th node, $g\left(\eta_{i}\right)=C \eta_{i}$ with $C \in R^{N \times N}$ being a matrix describing the dynamics of each single node, $D \in R^{m \times m}$ is the inner coupling matrix, and $G_{i j}$ is the adjacency matrix, $i, j=1, \ldots, N$.

Note that a solution of an isolated node satisfies

$$
\dot{s}(t)=g(s(t))
$$

where $s(t)=\left(s_{1}, \ldots, s_{m}\right)^{T}$ is the state vector. Here, only a linear model $g(s(t))=C s(t)$ is considered. For a general form of $g(s(t)), s(t)$ can be an equilibrium point, a periodic orbit, or even a chaotic orbit. For more details about synchronization in complex networks with nonlinear dynamics, see [17], [27], [29], [31], [32], [35], [37], [38]. The goal of synchronization in complex networks is to achieve $\lim _{t \rightarrow \infty}\left\|\eta_{i}(t)-\eta_{j}(t)\right\|=0, \forall i, j=$ $1, \ldots, N$.

Let $\eta=\left(\eta_{1}^{T}, \ldots, \eta_{N}^{T}\right)^{T}$ and rewrite system (6) into a matrix form

$$
\dot{\eta}(t)=\left[\left(I_{N} \otimes C\right)-c(L \otimes D)\right] \eta(t)
$$

Let $\Lambda=\operatorname{diag}\left(\lambda_{1}, \ldots, \lambda_{N}\right)$ be the diagonal matrix associated with $L$, i.e., there exists a unitary matrix $P$ such that $P^{T} L P=$ $\Lambda$. Then, one has

$$
\begin{aligned}
\left(P^{T}\right. & \left.\otimes I_{m}\right) \dot{\eta}(t) \\
& =\left[\left(P^{T} \otimes I_{m}\right)\left(I_{N} \otimes C\right)-c(\Lambda \otimes D)\left(P^{T} \otimes I_{m}\right)\right] \eta(t) \\
& =\left[\left(P^{T} \otimes C\right)-c(\Lambda \otimes D)\left(P^{T} \otimes I_{m}\right)\right] \eta(t) \\
& =\left[\left(I_{N} \otimes C\right)-c(\Lambda \otimes D)\right]\left(P^{T} \otimes I_{m}\right) \eta(t) .
\end{aligned}
$$

Let $x(t)=\left(P^{T} \otimes I_{m}\right) \eta(t)=\left(x_{1}^{T}, \ldots, x_{N}^{T}\right)^{T}$. Then, the above complex network model can be written as

$$
\dot{x}(t)=\left[\left(I_{N} \otimes C\right)-c(\Lambda \otimes D)\right] x(t)
$$

or

$$
\dot{x}_{i}(t)=\left(C-c \lambda_{i} D\right) x_{i}(t), i=1, \ldots, N
$$

Theorem 1: Suppose that the network $\mathcal{G}$ is connected. Synchronization in network (8) can be reached if and only if the following $N-1$ linear systems are asymptotically stable:

$$
\dot{x}_{i}(t)=\left(C-c \lambda_{i} D\right) x_{i}(t), i=2, \ldots, N .
$$

Proof: (Sufficiency). Since the network is connected, $1_{N} / \sqrt{N}$ is the unit eigenvector of the Laplacian matrix $L$ associated with the simple zero eigenvalue. From (9), one has $\eta(t)=\left(P \otimes I_{m}\right) x(t)$, where $L P=P \Lambda$ and $P=\left(p_{1}, \ldots, p_{N}\right)$. It is easy to verify that $p_{1}=1_{N} / \sqrt{N}$ is the unit eigenvector of the Laplacian matrix $L$ associated with the eigenvalue 0, i.e., $L p_{1}=0$. Since the $N-1$ systems in (10) are asymptotically stable, one has $\lim _{t \rightarrow \infty}\left\|x_{i}\right\| \rightarrow 0$ for $i=2, \ldots, N$. Therefore,

$$
\lim _{t \rightarrow \infty}\left\|\eta(t)-\frac{1}{\sqrt{N}}\left(x_{1}(t)^{T}, \ldots, x_{1}(t)^{T}\right)^{T}\right\|=0
$$

where $\dot{x}_{1}(t)=C x_{1}(t)$.

(Necessity). If synchronization in network (8) can be reached, then there exists a vector $\eta^{*}(t) \in R^{m}$ such that $\lim _{t \rightarrow \infty}\left\|\eta(t)-1_{N} \otimes \eta^{*}(t)\right\|=0$. Since $0_{N}^{T}=1_{N}^{T} L P=1_{N}^{T} P \Lambda=$ $\left(\lambda_{1} 1_{N}^{T} p_{1}, \ldots, \lambda_{N} 1_{N}^{T} p_{N}\right)$, one has $1_{N}^{T} p_{i}=0$ for $i=2, \ldots, N$. Therefore, $x_{i}(t)=\left(p_{i}^{T} \otimes I_{m}\right) \eta(t) \rightarrow\left(p_{i}^{T} 1_{N}\right) \otimes\left(I_{m} \eta^{*}(t)\right)=0$, as $t \rightarrow \infty$ for all $i=2, \ldots, N$. This completes the proof.

\section{B. The mth-Order Consensus in a General Form}

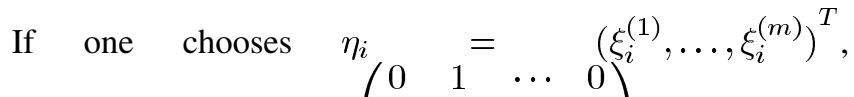

$$
\begin{aligned}
& C \quad\left(\begin{array}{cccc}
0 & 1 & \cdots & 0 \\
\vdots & \ddots & \ddots & \vdots \\
0 & 0 & \ddots & 1 \\
0 & 0 & \cdots & 0
\end{array}\right)_{m \times m}, \quad \text { and } \\
& D=\left(\begin{array}{cccc}
0 & 0 & \cdots & 0 \\
\vdots & \ddots & \cdots & 0 \\
\alpha_{1} & \alpha_{2} & \cdots & \alpha_{m}
\end{array}\right)_{m \times m} \text {, then the } m \text { th }
\end{aligned}
$$

protocol in multiagent systems (1) with the control input (5) is a special model of the complex network (6). Thus, the $m$ th-order consensus problem in system (1) with the control input (5) can be transformed to the synchronization problem $\lim _{t \rightarrow \infty}\left\|\eta_{i}(t)-\eta_{j}(t)\right\|=0, \forall i, j=1, \ldots, N$, in a network, which has just been discussed above.

Remark 1: In [22] and [24], second-order and third-order consensus in multiagent systems were studied by solving linear systems (8). It has been shown that second-order (third-order) consensus can be reached if and only if $\left(I_{N} \otimes C\right)-(L \otimes D)$ has exactly two (three) zero eigenvalues and all the other eigenvalues have negative real parts. Theorem 1 above generates the results in [22] and [24] to any positive integer $m$ by using a different method originated from synchronization in complex networks, i.e., employing the transverse stability to the synchronization manifold. In [7], the stability in cooperative networks with identical linear subsystems was considered where the equation with $i=1$, which is not in condition (10) of Theorem 1 here, also needs to be checked.

Corollary 1: Suppose that the network $\mathcal{G}$ is connected. The $m$ th-order consensus in the multiagent system (1) with protocol (5) can be reached if and only if the real parts of the roots in the following $N-1$ equations are all negative:

$$
\begin{array}{r}
\lambda^{m}+\alpha_{m} c \lambda_{i} \lambda^{m-1}+\cdots+\alpha_{2} c \lambda_{i} \lambda+\alpha_{1} c \lambda_{i}=0, \\
i=2, \ldots, N,
\end{array}
$$

i.e., polynomial (11) is stable. 
Proof: Let $\lambda$ be an eigenvalue of matrix $C-c \lambda_{i} D$. Then, one has $\operatorname{det}\left(\lambda I_{m}-C+c \lambda_{i} D\right)=0$. Note that

$$
\begin{aligned}
& \operatorname{det}\left(\lambda I_{m}-C+\lambda_{i} D\right) \\
& =\operatorname{det}\left(\begin{array}{cccc}
\lambda & -1 & \cdots & 0 \\
\vdots & \ddots & \ddots & \vdots \\
0 & 0 & \ddots & -1 \\
\alpha_{1} c \lambda_{i} & \alpha_{2} c \lambda_{i} & \cdots & \lambda+\alpha_{m} c \lambda_{i}
\end{array}\right)_{m \times m} \\
& =\lambda^{m}+\alpha_{m} c \lambda_{i} \lambda^{m-1}+\cdots+\alpha_{2} c \lambda_{i} \lambda+\alpha_{1} c \lambda_{i},
\end{aligned}
$$

where the last equation is obtained by determinantal expansion in the last row. Equation (10) is asymptotically stable if and only if the real parts of the roots in (11) are all negative. The proof is completed.

Remark 2: If $m=1$ and $\lambda=-\alpha_{1} c \lambda_{2}$, then first-order consensus can be achieved if and only if the network is connected [13], [23]. If $m=2$, then the real parts of the roots in $\lambda^{2}+$ $\alpha_{2} c \lambda_{i} \lambda+\alpha_{1} c \lambda_{i}=0$ are all negative if the network is connected and thus second-order consensus can be reached. However, for a general directed network, second-order consensus may fail even if the network contains a directed spanning tree [22]. In [33], some necessary and sufficient conditions for second-order consensus in multiagent dynamical systems with directed topologies were derived. Similarly, consensus in multiagent systems with specific higher order dynamics was discussed in [28]. However, in this paper, the results are based on a general framework, i.e., synchronization in complex networks, and consensus in multiagent systems with general identical linear subsystems (8) are studied. It should also be noted that the necessary and sufficient conditions in Theorem 1 and Corollary 1 can be used to study consensus in general directed networks where $\lambda_{i}$ are complex values, $i=2, \ldots, N$, as discussed in [28].

\section{Consensus Region in the mth-Order Consensus}

It is intuitively to see that Routh-Hurwitz criterion [3] can be applied to obtain a necessary and sufficient condition by solving the stability of (11) in Corollary 1 . However, this approach could result in the calculation of many high-order polynomial inequalities, which are very complicated and cannot be implemented for a general system.

Notice that it is generally difficult to check if (11) is stable for a large-scale network. As an alternative, let $\sigma>0$ be a variable and $\mathcal{S}=\left\{\sigma \mid \lambda^{m}+\alpha_{m} \sigma \lambda^{m-1}+\cdots+\alpha_{2} \sigma \lambda+\alpha_{1} \sigma=\right.$ 0 is stable $\}$ be the stable consensus region. Then, the problem is transformed to find if all the nonzero eigenvalues of the Laplacian matrix scaled by a factor $c$ lie in the stable consensus region $\mathcal{S}$. Consequently, the following criterion can be easily verified.

Corollary 2: Suppose that the network $\mathcal{G}$ is connected. The $m$ th-order consensus in the multiagent system (1) with protocol (5) can be reached if and only if

$$
c \lambda_{i} \in \mathcal{S}, i=2, \ldots, N \text {. }
$$

Let $\widetilde{P}(\lambda, \sigma)=\lambda^{m}+\alpha_{m} \sigma \lambda^{m-1}+\cdots+\alpha_{2} \sigma \lambda+\alpha_{1} \sigma$, where $\sigma \in\left[c \lambda_{2}, c \lambda_{N}\right]$. Therefore, if the polynomial $\widetilde{P}(\lambda, \sigma)$ is robustly stable for all $\sigma \in\left[c \lambda_{2}, c \lambda_{N}\right]$, the stability of all the polynomials in (11) can be obtained. Consequently, the following well-known Kharitonov's Theorem in robust control can be applied.

Proposition 1: (Kharitonov's Theorem [3]) Suppose that the network $\mathcal{G}$ is connected. The $m$ th-order consensus in the multiagent system (1) with protocol (5) can be reached if the four Kharitonov polynomials of $\widetilde{P}(\lambda, \sigma)$ with parameters $\sigma \in\left[c \lambda_{2}, c \lambda_{N}\right]$ are stable.

The computation of stability of four Kharitonov polynomials is very simple. However, the obtained condition in Proposition 1 is only sufficient since one needs to check if the polynomial $\widetilde{P}(\lambda, \sigma)$ is robustly stable for all $\sigma \in\left[c \lambda_{2}, c \lambda_{N}\right]$. Note that in Corollary 1 , in order to reach $m$ th-order consensus in the multiagent system (1), one only needs to check the stability of the polynomial $\widetilde{P}(\lambda, \sigma)$ at some discrete points $\sigma=c \lambda_{2}, \ldots, c \lambda_{N}$. In some particular cases, if the stable consensus regions are disconnected, the four Kharitonov polynomials may be unstable; however, all the nonzero eigenvalues of the Laplacian matrix scaled by a factor $c$ can still lie in these disconnected stable consensus regions.

In order to obtain a necessary and sufficient condition for reaching $m$ th-order consensus in the multiagent system (1), the objective next is to find the structure of the stable consensus region $\mathcal{S}$. In [6], [16], disconnected synchronization regions of complex networks were discussed. It was shown that there exist some disconnected synchronization regions in several particular complex networks when the synchronous state is an equilibrium point. In the following, a general $m$ th-order dynamical multiagent system (6) is considered, with corresponding criteria derived.

Lemma 2: Consider the polynomial

$$
P(\lambda)=\lambda^{m}+\alpha_{m} \sigma \lambda^{m-1}+\cdots+\alpha_{2} \sigma \lambda+\alpha_{1} \sigma,
$$

where $\alpha_{i}(i=1,2, \ldots, m)$ are constants. As $\sigma$ is varied, the sum of the orders of the zeros of $P(\lambda)$ on the open right-half plane can change only if a zero appears on or crosses the imaginary axis.

Proof: The zeros of $P(\lambda)$ continuously depend on the parameter $\sigma$. The result thus follows.

Lemma 3: $P(\lambda)=\lambda^{m}+\alpha_{m} \sigma \lambda^{m-1}+\cdots+\alpha_{2} \sigma \lambda+\alpha_{1} \sigma=0$ has a purely imaginary root if and only if

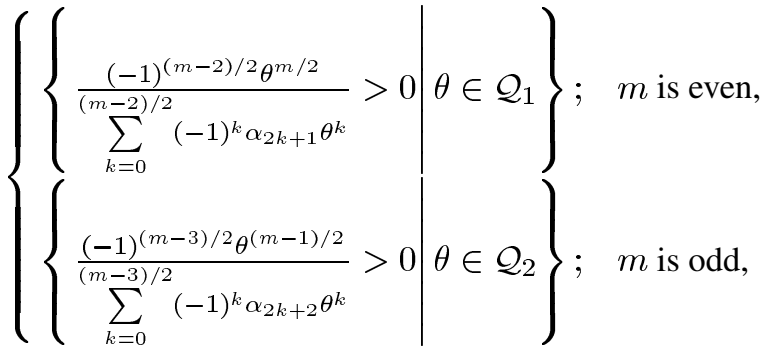

where $\mathcal{Q}_{1}=\left\{\theta>0 \mid \sum_{k=0}^{(m-2) / 2}(-1)^{k} \alpha_{2 k+2} \theta^{k}=0\right\}$ and $\mathcal{Q}_{2}=\left\{\theta>0 \mid \sum_{k=0}^{(m-1) / 2}(-1)^{k} \alpha_{2 k+1} \theta^{k}=0\right\}$.

Proof: It is easy to see that $P(\lambda)=0$ has $m$ zero roots if and only if $\sigma=0$. Let $\lambda=i \omega(\omega \neq 0)$. Then, one can show that $P(\lambda)=0$ has a purely imaginary root if and only if

$$
(i \omega)^{m}+\alpha_{m} \sigma(i \omega)^{m-1}+\cdots+\alpha_{2} \sigma(i \omega)+\alpha_{1} \sigma=0 .
$$


If $m=2 l$, where $l$ is a positive integer, then by separating the real and imaginary parts of (14), one obtains

$$
\left\{\begin{array}{l}
(-1)^{m / 2} \omega^{m}+\left[(-1)^{(m-2) / 2} \alpha_{m-1} \omega^{m-2}\right. \\
\left.+\cdots-\alpha_{3} \omega^{2}+\alpha_{1}\right] \sigma=(-1)^{m / 2} \omega^{m} \\
+\cdots \sum_{k=0}^{(m-2) / 2}(-1)^{k} \alpha_{2 k+1} \omega^{2 k}=0, \\
+-1)^{(m-2) / 2} \alpha_{m} \omega^{m-2}+(-1)^{(m-4) / 2} \alpha_{m-2} \omega^{m-4} \\
+\cdots-\alpha_{4} \omega^{2}+\alpha_{2}=\sum_{k=0}^{(m-2) / 2}(-1)^{k} \alpha_{2 k+2} \omega^{2 k}=0 .
\end{array}\right.
$$

Let $\mathcal{Q}_{1}=\left\{\theta>0 \mid \sum_{k=0}^{(m-2) / 2}(-1)^{k} \alpha_{2 k+2} \theta^{k}=0\right\}$. Then, $\mathcal{Q}_{1}$ has at most $(m-2) / 2$ elements. Thus, from (15), one obtains $\sigma=(-1)^{(m-2) / 2} \omega^{m} / \sum_{k=0}^{(m-2) / 2}(-1)^{k} \alpha_{2 k+1} \omega^{2 k}$.

If $m=2 l-1$, where $l$ is a positive integer, one can similarly get

$$
\left\{\begin{array}{l}
(-1)^{(m-1) / 2} \omega^{m}+\left[(-1)^{(m-3) / 2} \alpha_{m-1} \omega^{m-2}\right. \\
\left.+\cdots+\alpha_{2} \omega\right] \sigma=(-1)^{(m-1) / 2} \omega^{m} \\
+\sigma \omega \sum_{k=0}^{(m-3) / 2}(-1)^{k} \alpha_{2 k+2} \omega^{2 k}=0, \\
(-1)^{(m-1) / 2} \alpha_{m} \omega^{m-1}+(-1)^{(m-3) / 2} \alpha_{m-3} \omega^{m-3} \\
+\cdots-\alpha_{3} \omega^{2}+\alpha_{1}=\sum_{k=0}^{(m-1) / 2}(-1)^{k} \alpha_{2 k+1} \omega^{2 k}=0 .
\end{array}\right.
$$

The proof is thus completed.

From Lemma 3, one can see that there exist at most $\lfloor(m-$ $1) / 2\rfloor$ different positive values of $\sigma$ such that $P(\lambda)=0$ has a purely imaginary root, where $\lfloor(m-1) / 2\rfloor$ represents the integer part of the real number $(m-1) / 2$. Without loss of generality, suppose that there are $r$ different positive values, $0=\sigma_{0}<$ $\sigma_{1}<\cdots<\sigma_{r}<\sigma_{r+1}=\infty$, such that $P(\lambda)=0$ has a purely imaginary root if and only if $\sigma=\sigma_{i}$ for some $1 \leq i \leq r$ $(r \leq\lfloor(m-1) / 2\rfloor)$. Let $\mathcal{S}_{i}=\left(\sigma_{i-1}, \sigma_{i}\right), i=1, \ldots, r+1$.

Lemma 4: Suppose that the network $\mathcal{G}$ is connected. If there exists a positive value $\bar{\sigma} \in S_{i}$ such that $\lambda^{m}+\alpha_{m} \bar{\sigma} \lambda^{m-1}+\cdots+$ $\alpha_{2} \bar{\sigma} \lambda+\alpha_{1} \bar{\sigma}=0$ is stable (unstable), then, for any $\sigma \in \mathcal{S}_{i}=$ $\left(\sigma_{i-1}, \sigma_{i}\right), \lambda^{m}+\alpha_{m} \sigma \lambda^{m-1}+\cdots+\alpha_{2} \sigma \lambda+\alpha_{1} \sigma=0$ is stable (unstable), where $\lambda^{m}+\alpha_{m} \sigma \lambda^{m-1}+\cdots+\alpha_{2} \sigma \lambda+\alpha_{1} \sigma=0$ has a purely imaginary root when $\sigma=\sigma_{i}, i=1, \ldots, r$.

Proof: If $\lambda^{m}+\alpha_{m} \sigma \lambda^{m-1}+\cdots+\alpha_{2} \sigma \lambda+\alpha_{1} \sigma=0$ is stable (unstable), then all the roots of the polynomial have negative real parts (at least one root has a positive real part). By Lemma 3 , one knows that when $\sigma=\sigma_{i-1}$ or $\sigma=\sigma_{i}, P(\lambda)$ has a purely imaginary root. In view of Lemma 2 , the sum of the orders of the zeros of $P(\lambda)$ on the open right-half plane cannot change if $\sigma \in \mathcal{S}_{i}=\left(\sigma_{i-1}, \sigma_{i}\right)$. This completes the proof.

The positive real axis is now partitioned into $r+1$ intervals and $r$ points: $(0, \infty)=\mathcal{S}_{1} \bigcup\left\{\sigma_{1}\right\} \bigcup \cdots \bigcup\left\{\sigma_{r}\right\} \bigcup \mathcal{S}_{r+1}$. $P(\lambda)=0$ is not stable when $\sigma=\sigma_{i}, i=1, \ldots, r$. From Lemma 4 , in each interval $\mathcal{S}_{i}$, the sum of the orders of the zeros of $P(\lambda)$ on the open right-half plane or left-half plane remains the same for all $\sigma \in \mathcal{S}_{i}=\left(\sigma_{i-1}, \sigma_{i}\right), i=1, \ldots, r+1$.

Definition 2: $\mathcal{S}_{i}$ is called a stable consensus region if, for any $\sigma \in S_{i}, \lambda^{m}+\alpha_{m} \sigma \lambda^{m-1}+\cdots+\alpha_{2} \sigma \lambda+\alpha_{1} \sigma=0$ is stable; otherwise, $\mathcal{S}_{i}$ is called an unstable consensus region.
From Lemma 4, it is easy to see that the positive real axis is partitioned into several stable and unstable consensus regions. In [14], [16], the synchronization regions of complex networks are classified into three types: unbounded region, bounded region, and empty region. Here, in the $m$ th-order consensus problem, the stable consensus region can be any of these three types or even a union of them which may be composed of several disconnected stable consensus regions, as pointed out in [6].

Theorem 2: Suppose that the network $\mathcal{G}$ is connected. The $m$ th-order consensus in the multiagent system (1) with protocol (5) can be reached if and only if

$$
c \lambda_{i} \in \mathcal{S}, i=2, \ldots, N
$$

where $\mathcal{S}=\bigcup_{i \in \mathcal{N}} \mathcal{S}_{i}$ and $\mathcal{N}=\left\{i \mid \mathcal{S}_{i}\right.$ is a stable consensus region, $i=1, \ldots, r+1\}$.

Remark 3: For $m=1,2$, one can easily check that all the roots of $P(\lambda)=0$ have negative real parts, thus the stable consensus region is $(0, \infty)$. However, this may not be true for $m \geq$ 3. By computing $\sigma_{i}$, where $P(\lambda)=\lambda^{m}+\alpha_{m} \sigma_{i} \lambda^{m-1}+\cdots \overline{+}$ $\alpha_{2} \sigma_{i} \lambda+\alpha_{1} \sigma_{i}=0$ has a purely imaginary root as in Lemma 3, one can easily get $r+1$ disconnected regions, $\mathcal{S}_{i}=\left(\sigma_{i-1}, \sigma_{i}\right)$, $i=1, \ldots, r+1$. In view of Lemma 4 , stable and unstable consensus regions can be derived.

Corollary 3: Suppose that the network $\mathcal{G}$ is connected and $\mathcal{S}_{i}=\left(\sigma_{i-1}, \sigma_{i}\right)$ is a stable region for some $i, i=1, \ldots, r+1$. If $\left(\lambda_{N} / \lambda_{2}\right)<\left(\sigma_{i} / \sigma_{i-1}\right)$, then there exists a value $c>0$, such that $c \lambda_{j} \in \mathcal{S}_{i}$ for all $j=2, \ldots, N$. Thus, the $m$ th-order consensus in the multiagent system (1) with protocol (5) can be reached.

Proof: It suffices to prove that there exits a $c>0$ such that $\sigma_{i-1}<c \lambda_{2}$ and $c \lambda_{N}<\sigma_{i}$. Let $\left(\lambda_{N} / \lambda_{2}\right)+\varepsilon^{*}=\sigma_{i} / \sigma_{i-1}$, where $\varepsilon^{*}$ is a positive constant. One can choose $c^{*}>0$ such that $c^{*} \lambda_{2}=\sigma_{i-1}+\varepsilon$, where $\varepsilon$ is a sufficiently small positive value. From the condition $\left(\lambda_{N} / \lambda_{2}\right)<\left(\sigma_{i} / \sigma_{i-1}\right)$, one has $c^{*} \lambda_{N}<$ $\sigma_{i}-\varepsilon^{*} \sigma_{i-1}+\varepsilon\left(\sigma_{i} / \sigma_{i-1}-\varepsilon^{*}\right)$. Letting $\varepsilon$ be sufficiently small completes the proof.

Remark 4: If $\sigma_{i-1}=0$ or $\sigma_{i}=\infty$, then the condition $\left(\lambda_{N} / \lambda_{2}\right)<\left(\sigma_{i} / \sigma_{i-1}\right)$ is satisfied. The eigen-ratio $\lambda_{N} / \lambda_{2}$ can be considered as the consensus ability of the network. The smaller the $\lambda_{N} / \lambda_{2}$, the easier the network reaches consensus by choosing an appropriate value of $c$.

\section{LeAder-Follower CONTROL In Multiagent Systems}

In this section, the leader-follower control problem in multiagent systems is discussed. Assume that the leader evolves according to the following dynamics:

$$
\begin{aligned}
\dot{\xi}_{0}^{(1)}(t) & =\xi_{0}^{(2)}(t), \\
& \vdots \\
\dot{\xi}_{0}^{(m-1)}(t) & =\xi_{0}^{(m)}(t), \\
\dot{\xi}_{0}^{(m)}(t) & =u_{0},
\end{aligned}
$$

where $\xi_{0}^{(k)} \in R^{n}$ are the states of the leader and $u_{0}$ is a control input. 
In this subsection, the dynamics of all the followers, labeled $1, \ldots, N$, are governed by the multiagent system (1) with the control input

$$
\begin{aligned}
u_{i}(t) & =u_{0}+c \sum_{j=1, j \neq i}^{N} G_{i j} \sum_{k=1}^{m} \alpha_{k}\left(\xi_{j}^{(k)}(t)-\xi_{i}^{(k)}(t)\right) \\
& +c \beta_{i} \sum_{k=1}^{m} \alpha_{k}\left(\xi_{0}^{(k)}(t)-\xi_{i}^{(k)}(t)\right), i=1,2, \ldots, N,
\end{aligned}
$$

where $\beta_{i} \geq 0$ are the coupling strengths, $i=1,2, \ldots, N$. If $\beta_{i}>0$, then the follower $i$ can get the leader's information; if $\beta_{i}=0$, the leader's states are not available for the follower $i$. Therefore, only a small fraction of agents can sense the leader's information.

Let $e_{i}^{(j)}=\xi_{i}^{(j)}-\xi_{0}^{(j)}$ denote the relative error states to the leader, $e_{i}=\left(e_{i}^{(1)}, \ldots, e_{i}^{(m)}\right)^{T}$, and $e=\left(e_{1}^{T}, \ldots, e_{N}^{T}\right)^{T}$. Then, the error dynamics can be written as

$$
\dot{e}(t)=\left[\left(I_{N} \otimes C\right)-c(\widetilde{L} \otimes D)\right] e(t),
$$

where $\widetilde{L}=L+A$ and $A=\operatorname{diag}\left(\beta_{1}, \ldots, \beta_{N}\right)$.

Lemma 5: [5] If the network $\mathcal{G}$ is connected, $L_{i j}=L_{j i} \leq 0$ for $i \neq j$, and $\sum_{j=1}^{N} L_{i j}=0$, for all $i=1,2, \ldots, N$, then all eigenvalues of the matrix

$$
\left(\begin{array}{cccc}
L_{11}+\varepsilon & L_{12} & \cdots & L_{1 N} \\
L_{21} & L_{22} & \cdots & L_{2 N} \\
\vdots & \vdots & \ddots & \vdots \\
L_{N 1} & L_{N 2} & \cdots & L_{N N}
\end{array}\right)
$$

are positive for any constant $\varepsilon>0$.

From Lemma 5, one knows that $L+A$ is positive definite if the network $\mathcal{G}$ is connected and there is at least one positive diagonal element in $A$. Let $0<\mu_{1} \leq \cdots \leq \mu_{N}$ be the $N$ eigenvalues of $\widetilde{L}$.

Corollary 4: Suppose that the network $\mathcal{G}$ is connected. All agents in the multiagent system (1) with the control input (19) can follow the leader in (18) asymptotically if and only if the real parts of the roots in the following $N$ equations are all negative:

$$
\begin{array}{r}
\lambda^{m}+\alpha_{m} c \mu_{i} \lambda^{m-1}+\cdots+\alpha_{2} c \mu_{i} \lambda+\alpha_{1} c \mu_{i}=0, \\
i=1,2, \ldots, N .
\end{array}
$$

Proof: By following the same arguments as in Theorem 1 and Corollary 1 , the result can be easily proved.

Similar to Lemmas 3 and 4 , one can compute $\sigma_{i}$, where $\lambda^{m}+$ $\alpha_{m} \sigma \lambda^{m-1}+\cdots+\alpha_{2} \sigma \lambda+\alpha_{1} \sigma=0$ has a purely imaginary root when $\sigma=\sigma_{i}, i=1, \ldots, r$.

Theorem 3: Suppose that the network $\mathcal{G}$ is connected. All agents in the multiagent system (1) with the control input (19) can follow the leader in (18) asymptotically if and only if

$$
c \mu_{i} \in \mathcal{S}, i=1, \ldots, N,
$$

where $\mathcal{S}=\bigcup_{i \in \mathcal{N}} \mathcal{S}_{i}$ and $\mathcal{N}=\left\{i \mid \mathcal{S}_{i}\right.$ is a stable consensus region, $i=1, \ldots, r+1\}$.

From Theorems 2 and 3, it is easy to see that the computation of stable consensus regions in Lemma 2 plays a key role in the $m$ th-order consensus and leader-follower control of multiagent systems. The eigenvalues of $\widetilde{L}$ may lie in different stable regions. Since all the eigenvalues of $\widetilde{L}$ can be changed by choosing different followers, i.e., with different $i$ where $\beta_{i}>0$, it is desirable if all these eigenvalues $\mu_{i}$ lie in a particular stable region.

Corollary 5: Suppose that the network $\mathcal{G}$ is connected and $\mathcal{S}_{i}=\left(\sigma_{i-1}, \sigma_{i}\right)$ is a stable region for some $i, i=1, \ldots, r+1$. If

$$
\sigma_{i-1} I_{N}<c(L+A)<\sigma_{i} I_{N},
$$

then all agents in the multiagent system (1) with the control input (19) can follow the leader in (18) asymptotically.

Proof: If $\mathcal{S}_{i}$ is a stable region, then under condition (23), one has $c \mu_{1}>\sigma_{i-1}$ and $c \mu_{N}<\sigma_{i}$, which indicates that $c \mu_{j} \in$ $\mathcal{S}_{i}$ for all $j=1, \ldots, N$. This completes the proof.

Note that it is impossible for all agents to sense the leader in reality. To reduce the number of informed agents, some local feedback injections may be applied to a fraction of network nodes, which is known as pinning control [5], [35], [38]. It is still a challenging problem nowadays as how to choose the minimum number of informed agents such that (23) can be satisfied.

Corollary 6: Suppose that the network $\mathcal{G}$ is connected and $\mathcal{S}_{i}=\left(\sigma_{i-1}, \sigma_{i}\right)$ is a stable region for some $i, i=1, \ldots, r+1$. Under condition (23), it is necessary that

$$
\sigma_{i-1}<c L_{j j}
$$

holds for uninformed agents $j\left(\beta_{j}=0\right)$ and

$$
c L_{k k}<\sigma_{i}
$$

is satisfied for all agents, $k=1,2, \ldots, N$.

Proof: Under condition (23), one has $\sigma_{i-1}<c\left(L_{j j}+\right.$ $\left.\beta_{j}\right)<\sigma_{i}$ since every diagonal element of a positive definite matrix is positive. For uninformed agents $j$ with $\beta_{j}=0$, condition (24) is satisfied. For all agents, $c L_{j j} \leq c\left(L_{j j}+\beta_{j}\right)<\sigma_{i}$ hold. This completes the proof.

Remark 5: If one aims to choose some informed agents such that all the eigenvalues of $\widetilde{L}$ lie in a stable consensus region $\mathcal{S}_{i}=\left(\sigma_{i-1}, \sigma_{i}\right),(24)$ must hold for the uninformed agents, which shows that the agents with very small degrees $L_{j j}$ must be informed. For all agents, the maximum degree must be lower than $\sigma_{i} / c$; otherwise, some eigenvalues of $\widetilde{L}$ can not lie in $\mathcal{S}_{i}$. If $\mathcal{S}_{r+1}=\left(\sigma_{r}, \infty\right)$ is a stable consensus region, then by Corollary 5 , the condition (23) can be written as $c(L+A)-\sigma_{r} I_{N}>0$. If $c$ is sufficiently large, the $m$ th-order consensus can be reached by informing only one agent [5]. Some interesting schemes for choosing informed agents have been discussed in [35]. It was found that the nodes with low degrees should be informed first, which is contrary to the common view that the most-highly-connected nodes should be informed first. Furthermore, it has been shown that the derived pinning condition with leader's information given in a high-dimensional setting can be reduced to a low-dimensional condition without pinning controllers involved [35].

Remark 6: In many cases, it is impossible for an agent to measure all the states $\xi_{j}^{(k)}$ of its neighbors and the leader, $j=$ $0,1, \ldots, N ; k=1, \ldots, m$. For some order $k, \alpha_{k}=0$, which means that $\xi_{j}^{(k)}$ may be unavailable. In the second-order $(m=$ 2) leader-follower control problem of multiagent systems [9], 


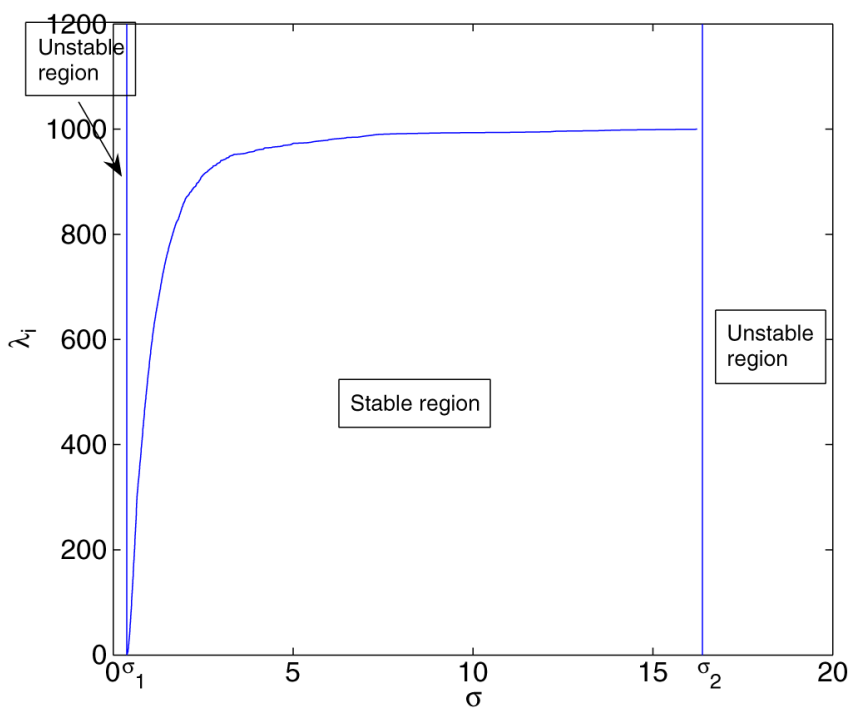

Fig. 1. Locations of $c \lambda_{i}, i=2, \ldots, N$.

[10], some observers were designed under the condition that the velocity states $\xi_{j}^{(2)}$ can not be measured by the agents. Here, a general higher order leader-follower control problem is considered. One natural question is whether Corollary 4 can still hold. Then, by designing an appropriate control input, similar results can also be obtained in [30, Sec. 4.3.2], which are omitted due to the limited page length.

\section{Simulation EXAmples}

In this section, some simulation examples are given to illustrate the theoretical results.

\section{A. Consensus Regions}

Consider the multiagent system (8) with $m=5, \alpha_{1}=1$, $\alpha_{2}=1.5, \alpha_{3}=4.16, \alpha_{4}=4$, and $\alpha_{5}=4$. From Lemma 3 , one has $\sigma_{1}=0.3816$ and $\sigma_{2}=16.3784$. By simple calculations, it is easy to verify that $\mathcal{S}_{2}=(0.3816,16.3784)$ is a stable consensus region. On regions $\mathcal{S}_{1}=(0,0.3816)$ and $\mathcal{S}_{3}=(16.3784, \infty), \lambda^{m}+\alpha_{m} \sigma \lambda^{m-1}+\ldots+\alpha_{2} \sigma \lambda+\alpha_{1} \sigma=0$, there are two eigenvalues with positive real parts and thus are unstable. It follows from Theorem 2 that the $m$ th-order consensus can be reached in system (8) if and only if $c \lambda_{i} \in(0.3816,16.3784)$ for all $i=2, \ldots, N$.

A scale-free network is performed in the simulation, where $N=1000$, the number of initial nodes is 5, and at each time step a new node is introduced and connected to 5 existing nodes in the network [2]. By computation, one obtains that $\lambda_{2}=2.8674$ and $\lambda_{N}=120.25$. From Corollary 3, one knows that $41.9369=$ $\left(\lambda_{N} / \lambda_{2}\right)<\left(\sigma_{2} / \sigma_{1}\right)=42.9203$. Therefore, by choosing $c=$ 0.135 , one has $0.3871=c \lambda_{2} \leq c \lambda_{i} \leq c \lambda_{N}=16.2338 \in$ $(0.3816,16.3784), i=2, \ldots, \bar{N}$. Thus, the $m$ th-order consensus of this network example in the form of the multiagent system (8) can be reached. The locations of $c \lambda_{i}$ are illustrated in Fig. 1 .

\section{B. Leader-Follower Control With Full-State Feedback}

Consider the multiagent system (19) with $m=5, c=0.8$, $\beta_{i}=30 \alpha_{1}=1, \alpha_{2}=2, \alpha_{3}=8, \alpha_{4}=4$, and $\alpha_{5}=5$.

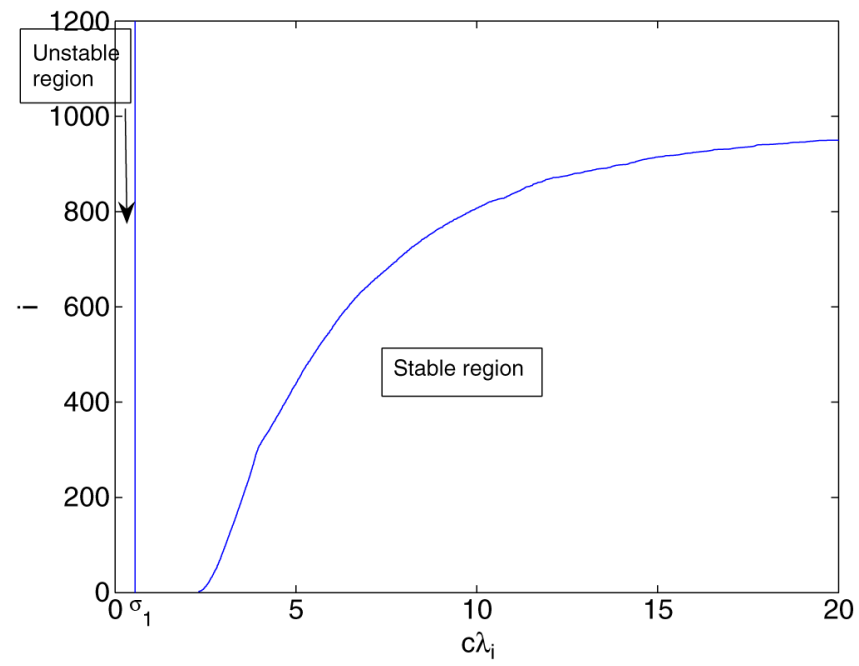

Fig. 2. Locations of $c \mu_{i}, i=1, \ldots, N$.

From Lemma 3, one has $\sigma_{1}=0.5557$. It is easy to verify that $\mathcal{S}_{2}=(0.5557, \infty)$ is a stable consensus region while $\mathcal{S}_{1}=$ $(0,0.5557)$ is unstable, where $\lambda^{m}+\alpha_{m} \sigma \lambda^{m-1}+\cdots+\alpha_{2} \sigma \lambda+$ $\alpha_{1} \sigma=0$ has two eigenvalues with positive real parts. The same scale-free network is simulated as above, assuming that there are 50 informed agents with the largest degrees, which can measure the information of the leader. In this case, one has $c \mu_{1}=0.6259>\sigma_{1}=0.5557$. Therefore, $c \mu_{i} \in \mathcal{S}_{2}$ for all $i=1, \ldots, N$. Thus, all agents in the multiagent system (19) can follow the leader in (18) asymptotically. The locations of $c \mu_{i}$ are illustrated in Fig. 2.

\section{CONCLUSIONS}

In this paper, general higher order distributed consensus protocols in multiagent dynamical systems have been studied. Some necessary and sufficient conditions have been derived for ensuring higher order consensus and it has been found that consensus can be reached if and only if all subsystems are asymptotically stable. Based on this result, consensus regions have been characterized, showing that consensus can be achieved if and only if all the nonzero eigenvalues of the Laplacian matrix lie in the stable consensus regions. It has also been found that the ratio of the largest to the smallest nonzero eigenvalues of the Laplacian matrix plays a key role in reaching consensus and a scheme for choosing an appropriate coupling strength has been derived. Finally, a leader-follower control problem in multiagent dynamical systems, has been studied, which reveals that the agents with very small degrees must be informed.

The distributed consensus protocols developed in this paper are very helpful for the design of cooperative control in multiagent dynamical systems, which could involve more complicated and realistic dynamics of autonomous mobile agents, for such as swarming and flocking of agents with higher order dynamics, time-varying and switching topologies, and nonidentical dynamics, leaving an interesting topic for future research. 


\section{REFERENCES}

[1] A. Arenas, A. Diaz-Guilera, J. Kurths, Y. Moreno, and C. Zhou, "Synchronization in complex networks," Phys. Rep., vol. 468, no. 3, pp. 93-153, 2008.

[2] A. L. Barabási and R. Albert, "Emergence of scaling in random networks," Science, vol. 286, pp. 509-512, 1999.

[3] B. R. Barmish, New Tools for Robustness of Linear Systems. New York: Macmillan, 1994.

[4] M. Cao, A. S. Morse, and B. D. O. Anderson, "Reaching a consensus in a dynamically changing environment: A graphical approach," SIAM J. Control Optim., vol. 47, no. 2, pp. 575-600, Feb. 2008.

[5] T. Chen, X. Liu, and W. Lu, "Pinning complex networks by a single controller," IEEE Trans. Circuits Syst. I, Reg. Papers, vol. 54, no. 6, pp. 1317-1326, 2007.

[6] Z. Duan, G. Chen, and L. Huang, "Disconnected synchronized regions of complex dynamical networks," IEEE Trans. Autom. Control, vol. 54, pp. 845-849, 2009.

[7] A. Fax and R. M. Murray, "Information flow and cooperative control of vehicle formations," IEEE Trans. Autom. Control, vol. 49, pp. 1465-1476, 2004.

[8] M. Fiedler, "Algebraic connectivity of graphs," Czechoslovak Math. J., vol. 23, pp. 298-305, 1973

[9] Y. Hong, G. Chen, and L. Bushnell, "Distributed observers design for leader-following control of multi-agent networks," Automatica, vol. 44, pp. 846-850, 2008.

[10] Y. Hong, J. Hu, and L. Gao, "Tracking control for multi-agent consensus with an active leader and variable topology," Automatica, vol. 42, pp. 1177-1182, 2006.

[11] R. A. Horn and C. R. Johnson, Matrix Analysis. Cambridge, U.K.: Cambridge Univ. Press, 1985.

[12] R. A. Horn and C. R. Johnson, Topics in Matrix Analysis. Cambridge, U.K.: Cambridge Univ. Press, 1991.

[13] A. Jadbabaie, J. Lin, and A. S. Morse, "Coordination of groups of mobile autonomous agents using nearest neighbor rules," IEEE Trans. Autom. Control, vol. 48, no. 6, pp. 985-1001, Jun. 2003.

[14] L. Kocarev and P. Amato, "Synchronization in power-law networks," Chaos, vol. 15, 2005, article no. 024101.

[15] Z. Li, Z. Duan, G. Chen, and L. Huang, "Consensus of multiagent systems and synchronization of complex networks: A unified viewpoint," IEEE Trans. Circuits Syst. I, Reg. Papers, vol. 57, no. 1, pp. 213-224, 2010.

[16] C. Liu, Z. Duan, G. Chen, and L. Huang, "Analyzing and controlling the network synchronization regions," Phys. A, vol. 386, pp. 531-542, 2007.

[17] J. Lü and G. Chen, "A time-varying complex dynamical network models and its controlled synchronization criteria," IEEE Trans. Autom. Control, vol. 50, no. 6, pp. 841-846, Jun. 2005.

[18] R. O. Saber and R. M. Murray, "Consensus problems in networks of agents with switching topology and time-delays," IEEE Trans. Autom. Control, vol. 49, no. 9, pp. 1520-1533, Sep. 2004.

[19] R. O. Saber, "Flocking for multi-agent dynamic systems: Algorithms and theory," IEEE Trans. Autom. Control, vol. 51, pp. 401-420, 2006.

[20] L. M. Pecora and T. L. Carroll, "Synchronization in chaotic systems," Phys. Rev. Lett., vol. 64, no. 8, pp. 821-824, 1990.

[21] W. Ren, "On consensus algorithms for double-integrator dynamics," IEEE Trans. Autom. Control, vol. 58, no. 6, pp. 1503-1509, 2008.

[22] W. Ren and E. Atkins, "Second-order consensus protocols in multiple vehicle systems with local interactions," presented at the AIAA Guidance, Navigation, Control Conf. Exhib., San Francisco, CA, 2005.

[23] W. Ren and R. W. Beard, "Consensus seeking in multiagent systems under dynamically changing interaction topologies," IEEE Trans. Autom. Control, vol. 50, no. 5, pp. 655-661, May 2005.

[24] W. Ren, K. L. Moore, and Y. Chen, "High-order and model reference consensus algorithms in cooperative control of multi-vehicle systems," ASME J.f Dyn. Syst., Meas., Control, vol. 129, no. 5, pp. 678-688, 2007.

[25] C. W. Reynolds, "Flocks, herds, and schools: A distributed behavior model," Comput. Graph., vol. 21, no. 4, pp. 25-34, Jul. 1987.

[26] T. Vicsek, A. Cziok, E. B. Jacob, I. Cohen, and O. Shochet, "Novel type of phase transition in a system of self-driven particles," Phys. Rev. Lett., vol. 75, no. 6, pp. 1226-1229, Aug. 1995.

[27] X. Wang and G. Chen, "Synchronization in scale-free dynamical networks: Robustness and fragility," IEEE Trans. Circuits Syst. I, Fundam. Theory Appl., vol. 49, pp. 54-62, Jan. 2002.
[28] P. Wieland, J. S. Kim, H. Scheu, and F. Allgöwer, "On consensus in multi-agent systems with linear higher-order agents," in Proc. 17th Word Congr. IFAC, 2008, pp. 1541-1546.

[29] C. Wu and L. O. Chua, "Synchronization in an array of linearly coupled dynamical systems," IEEE Trans. Circuits Syst. I, Fundam. Theory Appl., vol. 42, no. 8, pp. 430-447, Aug. 1995.

[30] W. Yu, "Multi-agent collective behaviors analysis and applications in complex networks and systems" Ph.D. dissertation, City Univ. Hong Kong, Hong Kong, China, 2010 [Online]. Available: http://www.ee. cityu.edu.hk/ wwyu/Thesis.pdf

[31] W. Yu, J. Cao, G. Chen, J. Lü, J. Han, and W. Wei, "Local synchronization of a complex network model," IEEE Trans. Syst., Man, Cybern. B, Cybern., vol. 39, no. 1, pp. 230-241, 2009.

[32] W. Yu, J. Cao, and J. Lü, "Global synchronization of linearly hybrid coupled networks with time-varying delay," SIAM J. Appl. Dyn. Syst., vol. 7, no. 1, pp. 108-133, 2008.

[33] W. Yu, G. Chen, and M. Cao, "Some necessary and sufficient conditions for second-order consensus in multi-agent dynamical systems," Automatica, vol. 46, no. 6, pp. 1089-1095, 2010.

[34] W. Yu, G. Chen, M. Cao, and J. Kurths, "Second-order consensus for multi-agent systems with directed topologies and nonlinear dynamics," IEEE Trans. Syst., Man, Cybern. B, Cybern., vol. 40, no. 3, pp. 881-891, 2010.

[35] W. Yu, G. Chen, and J. Lü, "On pinning synchronization of complex dynamical networks," Automatica, vol. 45, no. 2, pp. 429-435, 2009.

[36] W. Yu, G. Chen, Z. Wang, and W. Yang, "Distributed consensus filtering in sensor networks," IEEE Trans. Syst., Man, Cybern. B, Cybern., vol. 39, no. 6, pp. 1586-1577, 2009.

[37] J. Zhou, J. Lu, and J. Lü, "Adaptive synchronization of an uncertain complex dynamical network," IEEE Trans. Autom. Control, vol. 51, no. 4, pp. 652-656, 2006.

[38] J. Zhou, J. Lu, and J. Lü, "Pinning adaptive synchronization of a general complex dynamical network," Automatica, vol. 44, no. 4, pp. 996-1003, 2008.

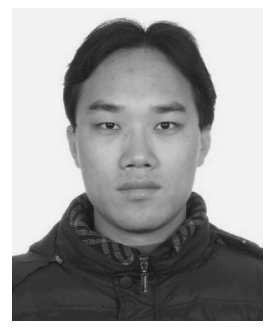

Wenwu Yu (S'07) received the B.Sc. degree in information and computing science and M.Sc. degree in applied mathematics from the Department of Mathematics, Southeast University, Nanjing, China, in 2004 and 2007, respectively, and the Ph.D. degree from the Department of Electronic Engineering, City University of Hong Kong, Hong Kong, China, in 2010.

He held several visiting positions in Australia, China, Germany, Italy, The Netherlands, and the United States. Currently, he is an Associate Professor in the Department of Mathematics, Southeast University. He is the author or coauthor of about 40 referred international journal papers, and a reviewer of several journals. His research interests include multiagent systems, nonlinear dynamics and control, complex networks and systems, neural networks, cryptography, and communications.

Prof. Yu is the recipient of the Best Master Degree Theses Award from Jiangsu Province, China in 2008, DAAD Scholarship from Germany in 2008, TOP 100 Most Cited Chinese Papers Published in International Journals in 2008, the Best Student Paper Award in 5th Chinese Conference on Complex Networks in 2009, and the First Prize of Scientific and Technological Progress Award of Jiangsu Province in 2010.

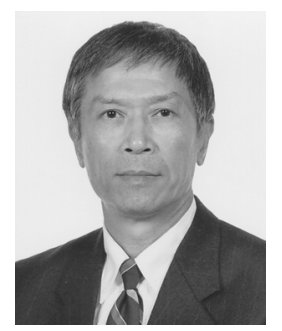

Guanrong Chen (M'89-SM'92-F'97) received the M.Sc. degree in computer science from Sun Yat-sen University, Guangzhou, China, in 1981 and the $\mathrm{Ph} . \mathrm{D}$. degree in applied mathematics from Texas A\&M University, College Station, in 1987.

Currently he is a Chair Professor and the Founding Director of the Centre for Chaos and Complex Networks at the City University of Hong Kong, prior to which he was a tenured Professor in the University of Houston, TX

Prof. Chen is currently Editor-in-Chief for the IEEE Circuits and Systems Magazine and for the International Journal of Bifurcation and Chaos. He received the 1998 Harden-Simons Prize for the Outstanding Journal Paper Award from the American Society of Engineering Education, the 2001 M. Barry Carlton Best Transactions Paper Award from the IEEE Aerospace and Electronic Systems Society, the 2002 Best Paper Award 
from the Institute of Information Theory and Automation, Academy of Science of the Czech Republic, and the 2005 IEEE Guillemin-Cauer Best Transaction Paper Award from the Circuits and Systems Society. He received the 2008 State Natural Science Award and the 2010 Ho-Leung-Ho-Lee Science and Technology Progress Award in China. He is Honorary Professor at different ranks in some thirty universities worldwide.

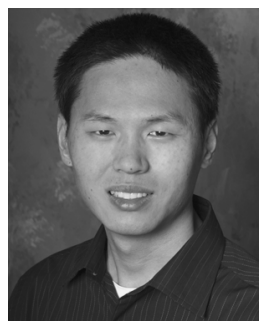

Wei Ren (S'01-M'04) received the B.S. degree in electrical engineering from Hohai University, China, in 1997, the M.S. degree in mechatronics from Tongji University, China, in 2000, and the Ph.D. degree in electrical engineering from Brigham Young University, Provo, UT, in 2004.

From October 2004 to July 2005, he was a Postdoctoral Research Associate with the Department of Aerospace Engineering, University of Maryland, College Park. Since August 2005, he has been with the Department of Electrical and Computer Engineering, Utah State University, Logan, where he is currently an Associate Professor. His research focuses on distributed control of multiagent systems, networked cyber-physical systems, and autonomous control of unmanned vehicles. He is an author of the books Distributed Coordination of Multi-agent Networks (Springer-Verlag, 2011) and Distributed Consensus in Multi-Vehicle Cooperative Control (Springer-Verlag, 2008).

Dr. Ren was a recipient of the National Science Foundation CAREER Award in 2008. He is currently an Associate Editor for Systems and Control Letters and an Associate Editor on the IEEE Control Systems Society Conference Editorial Board.

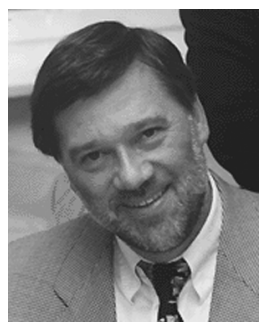

Jürgen Kurths studied mathematics at the University of Rostock and received the Ph.D. and Dr. habil. degrees at the GDR Academy of Sciences in 1983 and 1990, respectively.

He was a full Professor at the University of Potsdam from 1994 to 2008. Currently, he is a Professor of Nonlinear Dynamics at the Humboldt University, Berlin, Germany, and Chair of the research domain Transdisciplinary Concepts of the Potsdam Institute for Climate Impact Research. $\mathrm{He}$ has published more than 400 papers and two monographs which are cited more than 14000 times. His main research interests are complex synchronization phenomena, complex networks, time series analysis and their applications in climatology, sustainability, physiology and engineering.

$\mathrm{He}$ is a fellow of the American Physical Society and the Fraunhofer Society (Germany), and a member of Academia Europaea. He received a HumboldtCSIR research price in 2005 and a Dr. h.c. in 2008. He is in the editorial board of more than 10 journals, among them CHAOS, Philosophical Trans. Royal Soc. A, European J. Physics ST, and Nonlinear Processes in Geophysics.

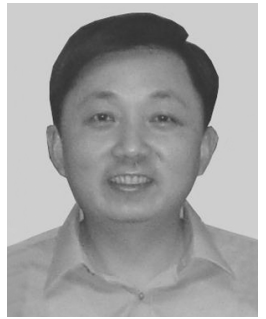

Wei Xing Zheng (M'93-SM'98) received the B.Sc. degree in applied mathematics and the M.Sc. and $\mathrm{Ph} . \mathrm{D}$. degrees in electrical engineering in 1982, 1984 and 1989, respectively, all from Southeast University, Nanjing, China.

$\mathrm{He}$ has held various faculty/research/visiting positions at Southeast University, China, Imperial College of Science, Technology and Medicine, U.K., University of Western Australia, Curtin University of Technology, Australia, Munich University of Technology, Germany, University of Virginia, and University of California-Davis. Currently he holds the rank of Full Professor at University of Western Sydney, Australia.

Dr Zheng has served on the technical program or organizing committee of numerous international conferences, including the 49th IEEE Conference on Decision and Control (CDC'2010), the 44th IEEE International Symposium on Circuits and Systems (ISCAS'2011), and the 16th IFAC Symposium on System Identification (SYSID'2012). He has also served on several IEEE or IFAC technical committees, and has been the Chair of IEEE Circuits and Systems Society's Technical Committee on Neural Systems and Applications and the Chair of IEEE Circuits and Systems Society's Technical Committee on Blind Signal Processing. He has served as an Associate Editor for four flagship journals: IEEE TRANSACTIONS ON CIRCUITS AND SYSTEMS-PART I: FUNDAMENTAL THEORY AND APPLICATIONS (2002-2004), IEEE TRANSACTIONS ON AUTOMATIC CONTROL (2004-2007), IEEE TRANSACTIONS ON CIRCUITS AND SYSTEMS-PART II: EXPRESS BRIEFS (2008-2009), and IEEE Signal Processing LETTERs (2007-2010). He also served as a Guest Editor of Special Issue on Blind Signal Processing and Its Applications for IEEE TRANSACTIONS ON CIRCUITS AND SYSTEMS-PART I: REGUlAR PAPERS (2009-2010). Since 2000, he has been an Associate Editor of the IEEE Control Systems Society's Conference Editorial Board. 